\title{
Analysis of Backward and Forward Effects on a Grooved Co-axial Heat Exchanger by Response Surface Methodology
}

\author{
Şahin GÜNGÖR ${ }^{* 1}$, Levent AYDIN ${ }^{1}$, Umut CEYHAN $^{1}$, Büşra KAYA $^{2}$, Ziya Haktan KARADENIZ \\ ${ }^{1}$ İzmir Katip Çelebi University, Faculty of Engineering and Architecture, Department of Mechanical Engineering, İZMiR \\ 2 İzmir Katip Çelebi University, Graduate School of Natural and Applied Sciences, İZMIR
}

\begin{abstract}
In the literature, it is proved that grooved pipe models are thermally more efficient than the smooth pipe model. Different than the previous studies in which the groove dimensions are constant along the pipe, we study the effect of groove radius and the gap between adjacent grooves on the local heat transfer coefficients using computational fluid dynamics software. The grooved section consists of three sub-sections to see the effects of groove dimension in stream-wise flow direction. We vary the radius of circular grooves parametrically in each section to optimize the local groove radius throughout the pipe. We couple the fluid flow $(1200<\operatorname{Re}<24000)$ with energy equations, and the grooved sections are set as heated wall at constant temperature of $350 \mathrm{~K}$. The optimal mesh has been selected by performing mesh independence study and finer mesh has been used in heated wall section. The radii of grooves are varied from 2 to $6 \mathrm{~mm}$ with an increment of $0.2 \mathrm{~mm}$ considering the manufacturability of the pipe, to do so we use the design of experiments (DOE). All DOE tools in ANSYS software are examined and compared with full factorial results. After DOE process, local heat transfer coefficient values of all groove parts are examined by response surface methodology (RSM).
\end{abstract}

\section{Introduction}

The grooved pipes are widely used in energy conversion devices such as heat pipes and heat exchangers to enhance the heat transfer. Both experimental and numerical methods are used to investigate the effect of geometric parameters of grooves on heat transfer. It can be assumed that early works on grooved pipe geometries have been initiated by Sparrow et al. [1,2]. Heat transfer, pressure drop and flow field visualization studies were performed experimentally for corrugated wall geometry in a range of $2000<\operatorname{Re}<33000$ and $4<\operatorname{Pr}<11$. They obtained that corrugation peaks brought a decrease in the Nusselt number at larger Reynolds numbers. Vicente et al. [3], studied experimentally on helical grooved pipe under laminar and transitional flow conditions. In the mixed convection analysis, the friction factor increased by $5 \%$ to $30 \%$ and the Rayleigh number increased by $30 \%$ compared to smooth pipe analysis. Experimental studies in which some kinds of groove geometries were examined in range of different Reynolds numbers [4-8] have made a great contribution to the literature. Experimental data obtained from these studies were compared with Dittus-Boelter and Gnielinski correlations. Eventually, it is proved that grooved pipe models have significant improvement on heat transfer compared with the smooth pipes. Bilen et al. [9] experimentally investigated rectangular, trapezoidal and circular grooved pipe models and found that the circular ones have the highest heat transfer coefficient.
With the development of computing technology and software abilities, the facility of studying in a wider range of issues has emerged and numerical studies have been accelerated. Wang et al. [10] investigated corrugation height and corrugation pitch on different type of heat exchangers with computational fluid dynamics (CFD) in a range of $1500<\mathrm{Re}<10000$. This study is important for that different corrugated plates proposed for different flow regions and they also examined the Nusselt number and friction factor variation depending on these plates. In addition, the results obtained with numerical analysis are also compared with the results of the experimental study [11] related to this topic in the literature. Several numerical and analytical methods have been used to design the optimal tube configuration to understand the effects of the parameters on heat transfer performance clearly. The numerical studies include design of experiments (DOE), response surface methodology (RSM) and optimization methods in addition to CFD analysis. Sun et al. [12] used CFD and RSM methods for elliptical finned tube heat exchangers. They defined the width/height ratio as an axis ratio for an elliptic shape and examined the variation of thermal performance with axis ratio. Central composite design (CCD) tool was used for DOE method and thermal performance of air and water sides were presented by response surface visualization. Han et al. [13] also defined dimensionless parameters as corrugation pitch, corrugation height etc. and performed the parametric analysis with CFD method.

Corresponding author: sahin.gungor@ikc.edu.tr 
Maximum Nusselt number was investigated with using the data set obtained from random search (RS) method. Wang et al. [14] compared the results of CFD analysis in range of high Re numbers (10020-40060) with similar experimental results [15]. The analysis for different corrugation heights and corrugation pitches were repeated and Nusselt number and friction factor graphs were presented. On the other hand, the variation of pressure drop and heat transfer coefficient values with external diameter, layer pitch and tube pitch were examined [16] using genetic aggregation and multi objective genetic algorithm (MOGA) for spiral heat exchanger. Neto et al. [17] investigated heat sink process with factorial design and RSM. Nookaraju et al. [18] handled helical grooved heat pipe model and obtained empirical relationship in terms of heat transfer coefficient with RSM. Mass flow rate of water, inclination angle and heat transfer values were inputs and maximum thermal performance were investigated.

Although there are many studies in the literature for different Reynolds numbers and grooved pipe models, the groove geometry was constant along the streamwise direction throughout the channel. However, in this numerical study, the local heat transfer performance for a coaxial and circular grooved pipe model is optimized by defining three independent sections with different groove diameters. An introductory example is initially solved in full factorial considering the criterion of manufacturability by additive manufacturing. Different Design of Experiments (DOE) algorithms (central composite, optimal design, box-behnken etc.) are tested to examine their validity. Backward-forward effect simulations and maximum-minimum heat transfer coefficient values are obtained with using Response Surface methodology for all scenarios. In addition, multiple nonlinear regression and optimization studies are performed in the Mathematica program. The aim of optimization study in Mathematica is to obtain the maximum the heat transfer coefficient of each groove sections and comparison of these values with RSM results. In the optimization process, Differential Evaluation, Simulated Annealing, Random Search, and Nelder-Mead algorithms have been used for the same problem to avoid inherent scattering of the stochastic search. Optimization algorithms are performed and the results are compared to reveal their abilities to capture the local changes.

\section{Material and Methods}

\subsection{Grooved Pipe Model}

The circular grooved pipe model that has three independent subsection and long inlet section is examined to maximize the heat transfer performance. Each section includes five grooves with constant radius within.

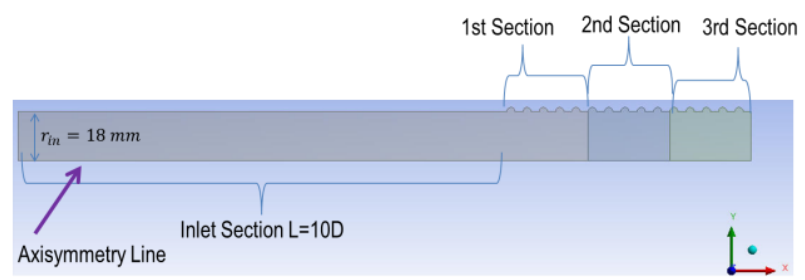

Fig.1. The computational domain.

A 2-dimensional axisymmetric model is used to reduce the computational load. The pipe has $36 \mathrm{~mm}$ diameter and the working fluid (air) enters at $298 \mathrm{~K}$ to the axisymmetric computational domain which has a long inlet section ( $\mathrm{L}=10 \mathrm{D})$ to provide fully developed flow conditions at the heat exchanger region. Reynolds number is between 1150 and 24000 at the inlet section. $\mathrm{K}-\varepsilon$ turbulence model with enhanced wall treatment is used at high Reynolds number $(\operatorname{Re}>2300)$ cases. The grooved sections are set as heated wall at constant temperature of $350 \mathrm{~K}$. Groove radii of each subsection are determined as independent inputs and the heat transfer coefficients of each grooved section (HTC1, HTC2 and HTC3) are defined as output parameters. Parametric studies are performed according to the manufacturable limits of additive manufacturing method. The radii of each grooved section have an increment of $0.2 \mathrm{~mm}$ between 2 to $6 \mathrm{~mm}$. Mesh size was decreased through the heated wall to obtain the correct results in CFD analyses, and the optimal mesh structure was selected by performing mesh independence studies.

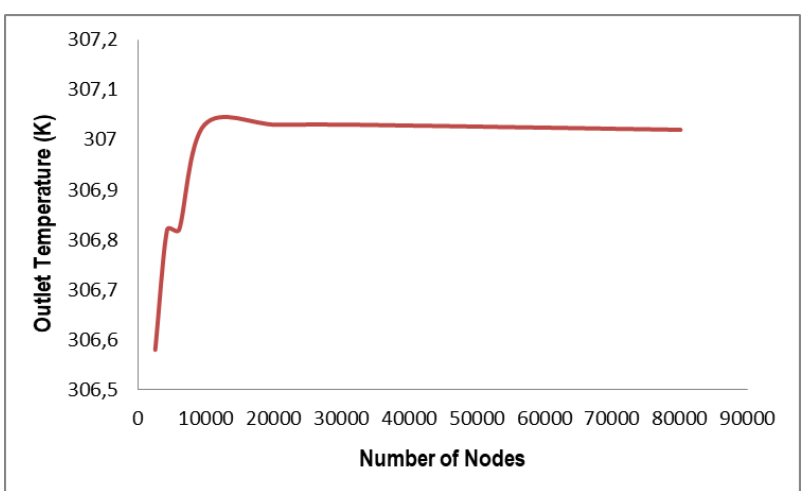

Fig.2. Mesh independence study.

\subsection{Optimization Study}

Optimization is the act of obtaining the best result under the given constraints. Determination of design variables affecting the phenomena, constraints of the system and objective function are the main steps of an adequate optimization study. Objective function can be obtained from analytical methods and data set gathered from experimental set-ups or numerical approaches. If there is no analytical equation to perform an optimization study, regression models are used to obtain an objective function that tells us the phenomena. In this study, radii of three grooved sections are determined as design variables. Considering the manufacturability of the model by additive manufacturing method, limits of the problem are set as constraint. Heat transfer coefficients of each grooved sections (HTC1, HTC2 and HTC3) are 
determined as outputs of the system under given physical conditions. Both full factorial and different numbers of scenarios in DOE methods are performed and data sets are obtained by numerical approaches. Data sets obtained from both full factorial design and DOE methods fed the response surface methodology (RSM) tool to obtain the best thermal results for different Reynolds numbers. Full factorial data set is also examined in Mathematica software by coding multiple nonlinear regression model and optimization process. The optimal results obtained from both RSM and Mathematica code are examined and compared by means of thermal performance of the system.

\subsubsection{DOE based RSM Method}

RSM illustrates the relation between the design variables and system outputs with regression fitting. RSM needs a data set, which can be created by both full factorial analyses and DOE approaches, to obtain the optimal responses. In this study, there are 9261 possible scenarios limited by the manufacturability criteria which are included in full factorial solution. Full factorial scenarios are analysed to compare the abilities of DOE algorithms and Mathematica optimization coding. Since solving of whole possible scenarios is not an efficient way, DOE algorithms are used to illustrate the phenomena with less experiments (design point). DOE is a statistical approach and technique used for guiding the choice of the design points in an efficient way [19]. DOE method includes these steps;

- Determination of the problem and phenomena,

- Determination of the parameters, factors or inputs that effecting the phenomena,

- Decision of the system outputs,

- Implementation of the constraints or limits,

- Determination of DOE algorithm,

-Obtaining the DOE results depending on design points,

Firstly, full factorial scenarios are analysed by CFD software of ANSYS Fluent for different Reynolds numbers. After that the sub tools of DOE in ANSYS, which are central composite design, optimal design, sphare grid initialization, custom and sampling, box behnken and latin hypercube design, are examined and compared with full factorial results. The aim in the comparison of full factorial and different DOE technique results is that there are too much time, computer and human source waste for full factorial analyses and DOE gives an opportunity to understand the correct phenomena with less effort.

The main idea is to use the results of a DOE run in order to create an approximation of the response variable over the design space [19], so we can say that DOE techniques are used for collecting data that is needed in RSM. We can obtain minimum and maximum values of output parameters in RSM. Therefore, RSM method is performed for processing the data set and simulating the variation of the local heat transfer coefficient of each grooved sections. Figure 3 shows the number of experiments $(\mathrm{N})$ required by different DOE algorithms depending on the number of parameters $(\mathrm{k})$. In this study, there are three input parameters and $\mathrm{N}<20$ for each DOE technique in compatible with the reference graphic. The data sets gathered from both full factorial and DOE methods are transported to response surface tool to obtain an illustration between design variables and responses.

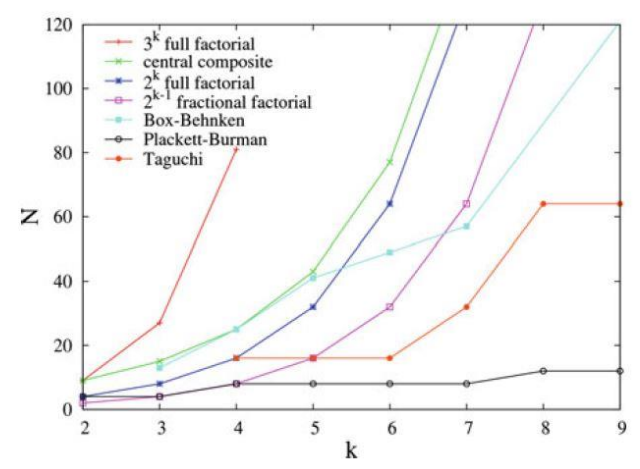

Fig.3. Number of experiments required by the DOE techniques [19].

\subsubsection{Stochastic Algorithms}

In this study, local heat transfer coefficient of the three grooved sections (HTC1, HTC2 and HTC3) are analysed with CFD software and data set obtained for full factorial and all DOE scenarios. $2^{\text {nd }}$ order nonlinear rational regression model is chosen in Mathematica software to present the phenomena.

Second order rational $=\frac{a_{0}+a_{1} \cdot x_{1}+a_{2} \cdot x_{2}+a_{3} \cdot x_{1} \cdot x_{2}+a_{4} \cdot x_{1}^{2}+a_{5} \cdot x_{2}^{2}}{b_{0}+b_{1} \cdot x_{1}+b_{2} \cdot x_{2}+b_{3} \cdot x_{1} \cdot x_{2}+b_{4} \cdot x_{1}^{2}+b_{5} \cdot x_{2}^{2}}$

$\mathrm{R}$ square and adjusted $\mathrm{R}$ square values are calculated over 0.97 . Stability analyses are also performed to check the regression model for intermediate values that does not take place in data set. After stability control, we have an objective function that covers the design points and gives us the correct local heat transfer coefficient values. Most of the engineering systems including nonlinearity has discrete spaces, stochastic optimization algorithms gives more accurate results in contrast to deterministic algorithms. Because the stochastic optimization algorithms are derivative free and more reliable, in this study four stochastic optimization algorithms of Differential Evolution (DE), modified Nelder-Mead (NM), Random Search (RS) and Simulating Annealing (SA) are examined in terms of local heat transfer. These stochastic optimization algorithms are performed and results are compared with the full factorial and DOE results in ANSYS. Differential Evolution is an iterative optimization method, which is based on a genetic algorithm in terms of its operation. This optimization can be thought of as the survival of genetically strong, well characterized individuals, or the selection of them to generate new group. 


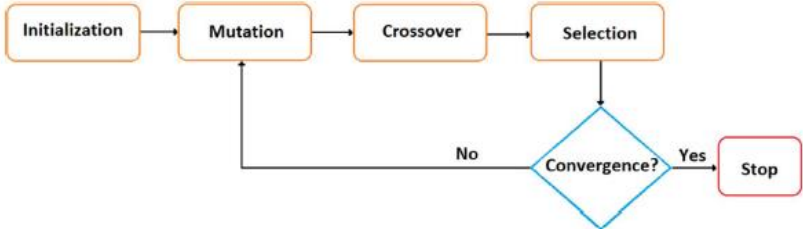

Fig.5. Flowchart of the DE algorithm [20].

The modified Nelder Mead (NM) algorithm, also known as Simplex, is a traditional local search method. Similar to that of DE algorithm, the adjustment of the NM options is controlled by four basic parameters: reflection, expansion, contraction, and shrinkage.

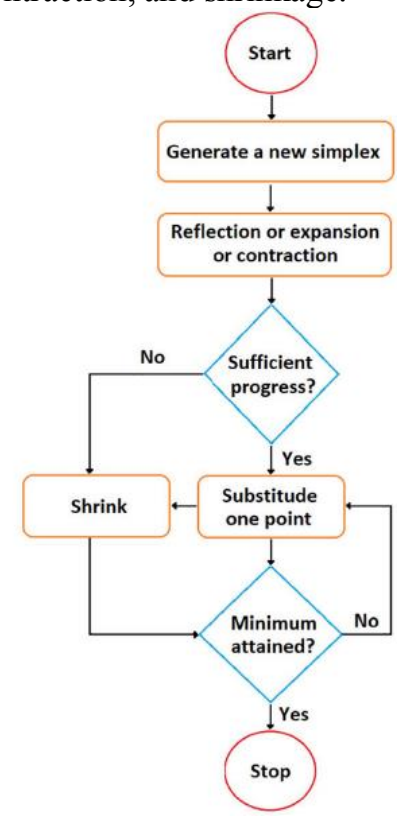

Fig. 6. Flowchart of the NM algorithm [20].

$\mathrm{RS}$ is a kind of a widely-used nondeterministic direct search method which also known as easy to adopted to complex problems. This method is an algorithm that achieves the optimum result depending on the number of iterations. The iterations start from an initial search point or set of points, to search for accepting random points in the search space until it reaches the optimum result.

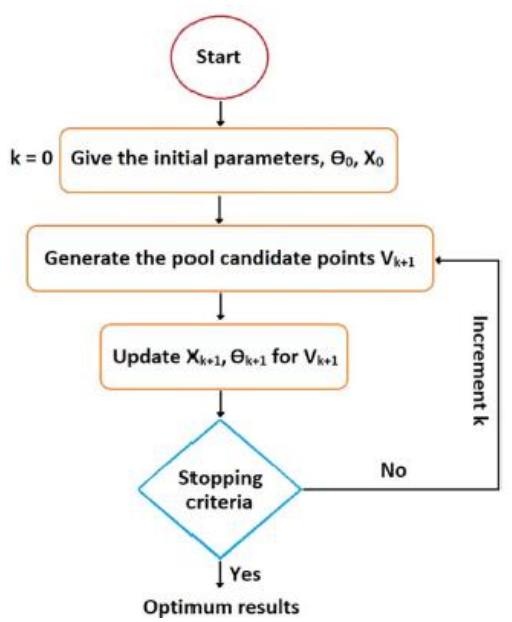

Fig. 7. Flowchart of the RS algorithm [20].
Simulated Annealing is based on the annealing process because of the way the algorithm works. As known, the simulated annealing algorithm is a random search technique that copies the physical process of annealing.

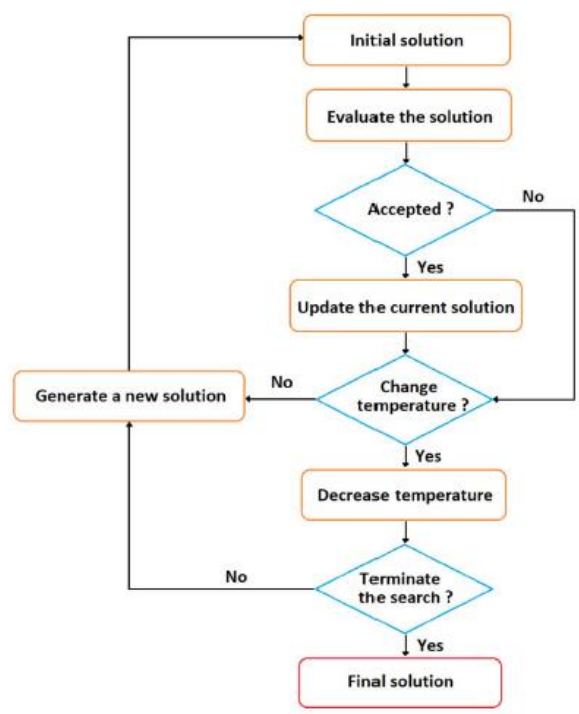

Fig. 8. Flowchart of the SA algorithm [20].

\section{Results and Discussion}

\subsection{Results of full factorial and DOE algorithm analyses}

Reynolds number is varied between approximately 1200 to 24000 for clearly observing the laminar and turbulence flow regions on circular grooved model. Velocity contours of each Reynolds number scenarios (Fig. 9) are examined in terms of being fully developed.

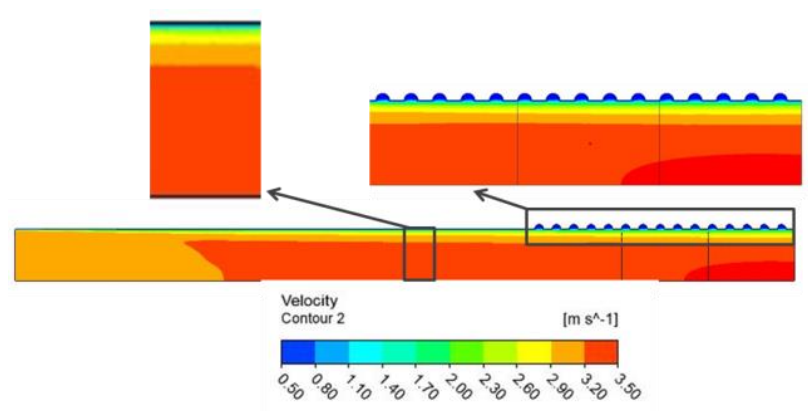

Fig.9. Sample of velocity contour $(3 \mathrm{~m} / \mathrm{s}$ inlet velocity, $\mathrm{Re}=6696$ ).

Table 1 shows the heat transfer coefficient results for the data set of full factorial and DOE algorithms. Minimum and maximum values of HTC1, HTC 2 and HTC 3 proves that heat transfer coefficient of each grooved section can enhance at a rate of approximately $20 \%$. In addition to this issue it can be deduced that DOE results of different algorithms are very close to both each other and the results of full factorial case. This is very critical result, because we decided that capability of DOE techniques is sufficient in terms of the determination of the phenomena with less effort. 
Table 1. HTC results of grooved sections for full factorial and DOE algorithms $(\mathrm{Re}=2464)$.

\begin{tabular}{|c|c|c|c|c|c|c|}
\hline \multirow[b]{2}{*}{ METHODS } & \multicolumn{2}{|c|}{ HTC1 } & \multicolumn{2}{|c|}{ HTC2 } & \multicolumn{2}{|c|}{ HTC3 } \\
\hline & Min. & Max. & Min. & Max. & Min. & Max. \\
\hline Full Factorial & 8.535 & 10.424 & 5.691 & 6.753 & 5.025 & 5.929 \\
\hline $\begin{array}{c}\text { DOE (Central } \\
\text { Composite) }\end{array}$ & 8.572 & 10.435 & 5.765 & 6.6413 & 5.062 & 5.858 \\
\hline $\begin{array}{l}\text { DOE (Optimal } \\
\text { Design) }\end{array}$ & 8.0983 & 10.422 & 5.667 & 6.767 & 5.061 & 5.868 \\
\hline $\begin{array}{c}\text { DOE (Sphare Grid } \\
\text { Initilization) }\end{array}$ & 8.579 & 10.425 & 5.755 & 6.723 & 5.076 & 5.882 \\
\hline $\begin{array}{l}\text { DOE (Custom and } \\
\text { Sampling) }\end{array}$ & 8.452 & 10.451 & 5.735 & 6.729 & 5.108 & 5.868 \\
\hline $\begin{array}{c}\text { DOE (Box-Behnken } \\
\text { Design) }\end{array}$ & 8.579 & 10.405 & 5.688 & 6.669 & 5.101 & 5.875 \\
\hline $\begin{array}{c}\text { DOE (Latin } \\
\text { Hypercube Design) }\end{array}$ & 8.467 & 10.429 & 5.747 & 6.728 & 5.055 & 5.889 \\
\hline
\end{tabular}

Figure 10 presents the maximum and minimum DOE results in terms of heat transfer coefficient of each grooved sections depending on different Reynolds numbers.
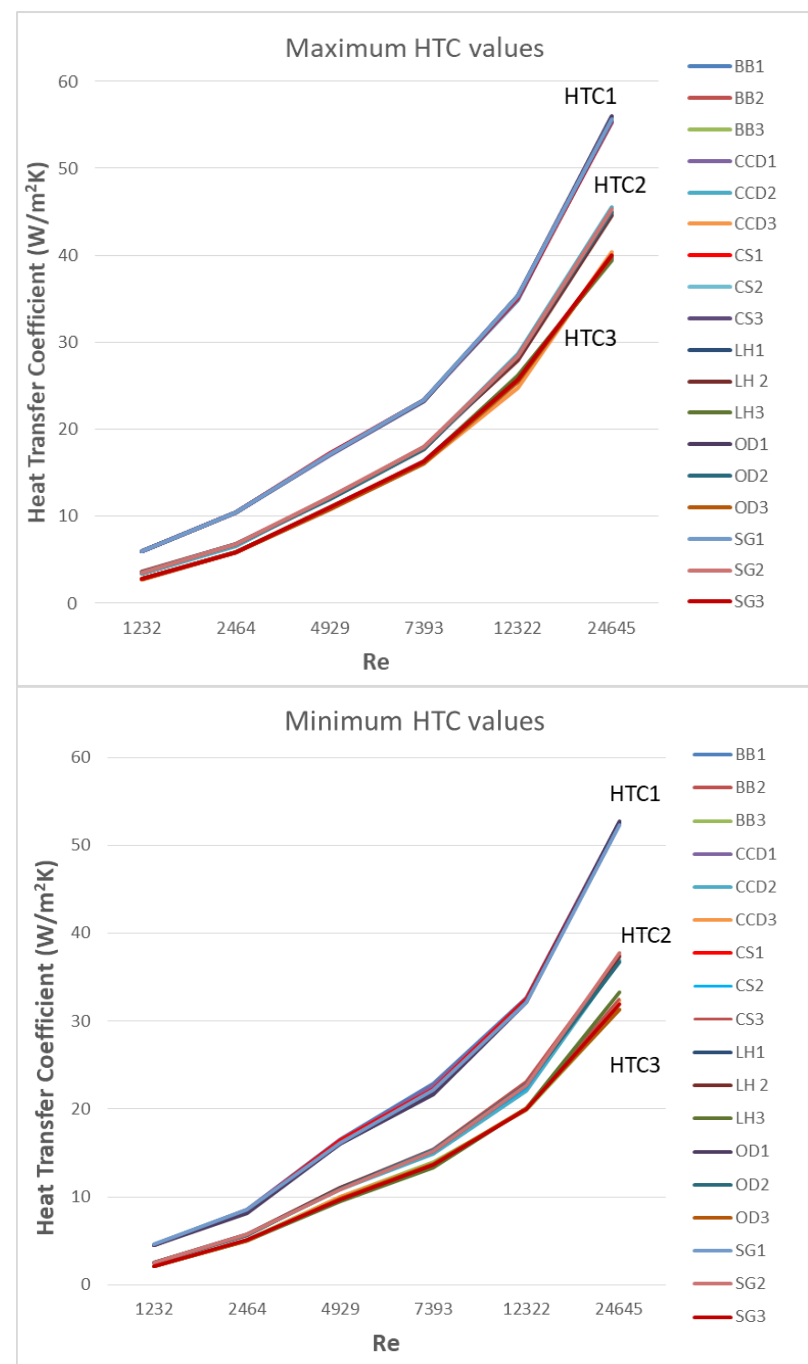

Fig. 10. Max. and min. heat transfer coefficient (HTC) values for each grooved section; BB: Box-Behnken, CCD: Central Composite Design, CS: Custom and Sampling, LH: Latin Hypercube, OD: Optimal Design, SG: Sparse Grid Initialization and 1, 2 and 3 presents the HTC1, HTC2 and HTC3, respectively.
Ability of DOE algorithms for both laminar and turbulence flow regions are examined and the results are presented in Figure 10. All DOE tools assigned design points between 15 and 20 in conformity with the number of experiment given in Figure 3. The results show that all DOE algorithms are successful to present the phenomena and there exist only a little difference between the techniques in low Reynolds numbers. However, the HTC values obtained from different DOE algorithms are getting to vary at high Reynolds number $(\operatorname{Re}>20000)$. This proves that if the Reynolds number or nonlinearity in the problem increase, the DOE results may vary in terms of fitting the phenomena.

\subsection{Outputs of the Optimization Study}

The main aim of the optimization study in Mathematica is the comparison the optimal local heat transfer coefficient values with full factorial data set obtained by CFD solver. Stochastic optimization algorithms of Differential Evolution (DE), modified Nelder-Mead (NM), Random Search (RS) and Simulating Annealing (SA) are examined in terms of local heat transfer performance. Statistical and mathematical approaches are performed to obtain an adequate regression model (objective function) in terms of fitting the full factorial data. $R$ square, adjusted $R$ square values are calculated in Mathematica software for each trial. Second order rational regression model is chosen as objective function because R square, adjusted R square values (over 0.97) and stability control results are good enough. Most optimization study is inadequate due to they don't examine the stability of the regression model. In this study, regression model is tested for intermediate values in the design point data set and stability of the model is checked.

Table 2. Stability control tests of nonlinear regression model.

\begin{tabular}{|c|c|c|c|c|}
\hline $\begin{array}{c}\mathbf{g r 1} \\
(\mathbf{m m})\end{array}$ & $\begin{array}{c}\mathbf{g r 2} \\
(\mathbf{m m})\end{array}$ & $\begin{array}{c}\mathbf{g r 3} \\
(\mathbf{m m})\end{array}$ & $\begin{array}{c}\text { HTC1 } \\
\left(\mathbf{W} / \mathbf{m}^{\wedge} \mathbf{2 . K}\right)\end{array}$ & $\begin{array}{c}\text { Model Result } \\
\left(\mathbf{W} / \mathbf{m}^{\wedge} \mathbf{2 . K}\right)\end{array}$ \\
\hline 2 & 2 & 2 & 10.366 & \\
\hline 2.2 & 2 & 2 & 10.213 & \\
\hline 2.1 & 2 & 2 & & 10.27449772 \\
\hline 3.4 & 2.4 & 2 & 9.6092 & \\
\hline 3.6 & 2.4 & 2 & 9.576 & \\
\hline 3.433 & 2.4 & 2 & & 9.571788617 \\
\hline 5 & 2.8 & 2 & 9.0197 & \\
\hline 5,2 & 2.8 & 2 & 8.6912 & \\
\hline 5.1421 & 2.8 & 2 & & 8.803309442 \\
\hline
\end{tabular}

Table 2 shows the stability tests of $2^{\text {nd }}$ order rational regression model, where gr 1 , gr 2 and gr 3 are the grove radius of the first, second and third sections, respectively. The check points given in Table 2 are only samples and in fact stability tests are performed in detailed with lots of intermediate unknown points. The results proved that $2^{\text {nd }}$ order rational regression model is very successful and stabile in term of fitting the data set. 
Optimization studies are performed as soon as the decision of accurate objective function by regression model and stability control tests. Objective function and the manufacturability constraints are defined as Mathematica codes and the optimal results for each grooved section are gathered.

Table 3. Optimization results in Mathematica software for $\mathrm{Re}=1240$.

\begin{tabular}{|c|c|c|c|c|}
\cline { 3 - 5 } \multicolumn{2}{c|}{} & HTC1 & HTC2 & HTC3 \\
\hline \multirow{2}{*}{ RSM } & Min. & 8.535 & 5.691 & 5.025 \\
\cline { 2 - 5 } & Max. & 10.424 & 6.753 & 5.929 \\
\hline \multirow{2}{*}{$\begin{array}{c}\text { Optimization } \\
\text { Mathematica }\end{array}$} & Min. & 8.564 & 5.715 & 5.091 \\
\cline { 2 - 5 } & Max. & 10.381 & 6.724 & 5.892 \\
\hline \multirow{2}{*}{ Difference (\%) } & Min. & -0.339 & -0.437 & -1.313 \\
\cline { 2 - 5 } & Max. & 0.412 & 0.427 & 0.624 \\
\hline
\end{tabular}

Full factorial data set obtained from CFD solver is transferred to Mathematica software and optimization steps are coded to obtain the best thermal results with stochastic optimization algorithms. Maximum heat transfer coefficients of each grooved section given in Table 3 are very close to optimal RSM data. We obtained the same results by all stochastic optimization algorithms; however Nelder-Mead is the fast optimization algorithm to obtain the optimal grove radius points.

\subsection{Results of Backward and Forward effect}

This study also examines the backward and forward effects of the grooved section radii in response surface optimization (RSM) tool in ANSYS. Since the RSM is the second step of DOE method to attain the correct response of the inputs and it scans area of the DOE design points more detailed, we used this method to observe the local thermal effects. Early results showed that the optimal points for local heat transfer coefficients nonlinearly changes with Reynolds number. The circular grooves have not so much thermal effect for low Reynolds numbers $(<3000)$ since pseudo laminar behaviour of the flow. The flow is not able to penetrate the grooves because of laminar behaviour in low Reynolds numbers. Therefore, we obtained the maximum heat transfer coefficients in the case that the groove radii are minimum (Table 4).

Table 4. Maximum and minimum heat transfer coefficient (HTC) values $(\mathrm{Re}=1240)$

\begin{tabular}{|c|c|c|c|c|c|}
\multicolumn{2}{c}{} & $\begin{array}{c}\text { 1st groove } \\
\text { radius } \\
(\mathbf{m m})\end{array}$ & $\begin{array}{c}\text { 2nd groove } \\
\text { radius } \\
(\mathbf{m m})\end{array}$ & $\begin{array}{c}\text { 3rd groove } \\
\text { radius } \\
(\mathbf{m m})\end{array}$ & $\begin{array}{c}\text { HTC } \\
\left(\mathbf{W} / \mathbf{m}^{\wedge} \mathbf{2 . K}\right)\end{array}$ \\
\hline \multirow{2}{*}{$\begin{array}{c}\text { 1st } \\
\text { section }\end{array}$} & Min. & 6 & 2 & 2 & $\mathbf{8 . 6 4 1 8}$ \\
\cline { 2 - 6 } & Max. & 2 & 4.8 & 5.2 & $\mathbf{1 0 . 4 0 2 0}$ \\
\hline \multirow{2}{*}{$\begin{array}{c}\text { 2nd } \\
\text { section }\end{array}$} & Min. & 3.4 & 6 & 2 & $\mathbf{5 . 7 4 2 4}$ \\
\cline { 2 - 6 } & Max. & 6 & 2 & 5.4 & $\mathbf{6 . 6 9 8 3}$ \\
\hline \multirow{2}{*}{$\begin{array}{c}\text { 3rd } \\
\text { section }\end{array}$} & Min. & 2.2 & 4.2 & 4.6 & $\mathbf{5 . 1 2 4 9}$ \\
\cline { 2 - 6 } & Max. & 2.4 & 5.2 & 2 & $\mathbf{5 . 8 6 0 3}$ \\
\hline
\end{tabular}

In contrast to that when the Reynolds number and the turbulence quantity increased thermal effects of grooved sections also increased. The local heat transfer coefficient of a grooved section is not only affected the own groove radius but also the groove radii of backward and forward sections. The local effects can be illustrated with response surfaces for being understood clearly. Figure 11 represents a sample of backward and forward effect examination with RSM tool.
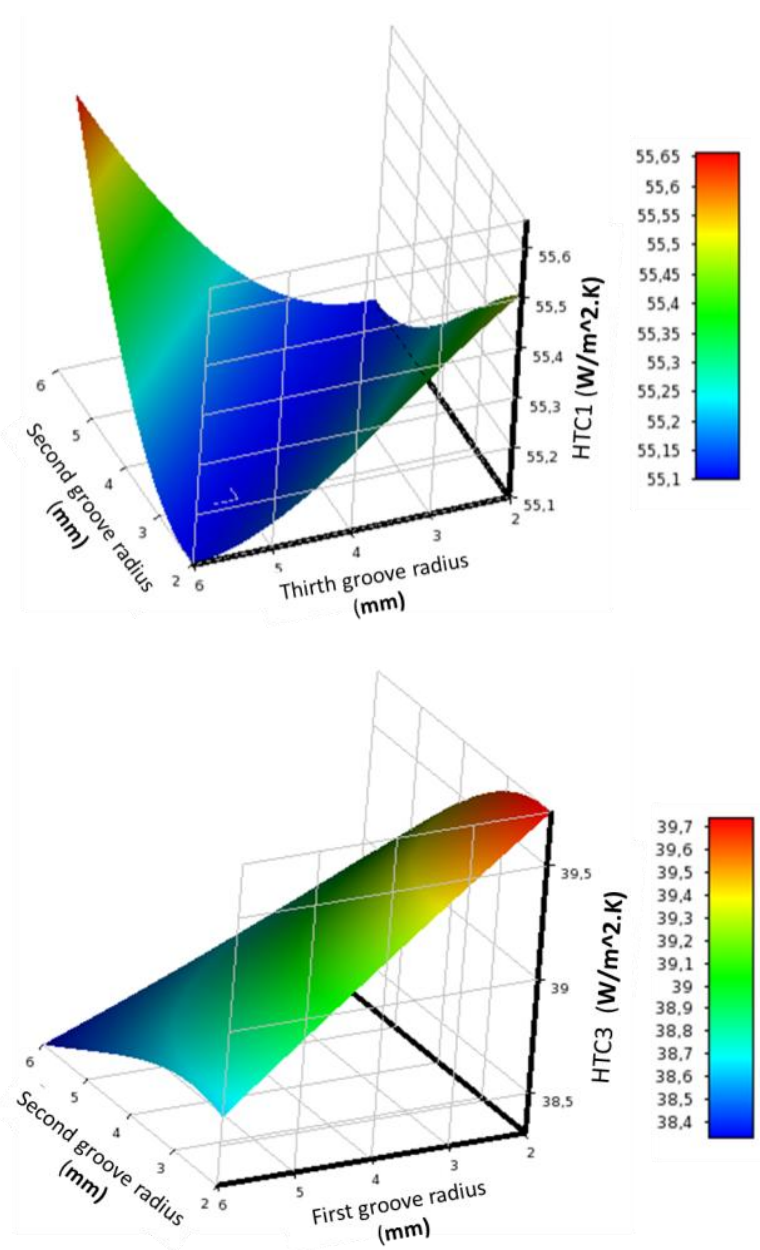

Figure 11. Sample of a backward effect (up) and forward effect analysis in RSM ( $\operatorname{Re} \sim 12000)$

Figure 11 shows that, local heat transfer quantity can be enhanced by variation of the previous and next grooved radii. Response surfaces topology also changes with the Reynolds number since the groove effects are dominant when the flow is turbulent. So, we analysed and obtained response surfaces in large range (approx. 1200 to 24000). The results showed that local heat transfer coefficient values can vary with the thermal performance at backward and forward sections. Forward effect is more impressive than backward effect, because it determines the flow distribution and velocity field dominantly. Response surfaces about backward and forward effect in Fig. 11 are valid for turbulent flow at a Reynolds number of 12000 . Response surfaces presents the local maximum heat transfer coefficients are executed when the backward groove radii are maximum or forward groove radii are minimum. 


\section{CONCLUSION}

Optimization and enhancement processes are aimed at making the current systems more efficient in terms of economic, energetic, strength etc. and numerous academic and industrial studies are being investigated on those issues. At this point especially in optimization studies, infinite number of scenarios can emerge, however the DOE concept can alleviate this burden. Considering that computer load and consumption are parameter when optimizing systems, we can easily determine the phenomena with DOE and reduce the computational load. This study is a basic guide for optimization of thermal-fluid engineering systems and also includes a sample application for systems having local heat transfer requirements. The results show that both optimization studies with mathematical codes and numerical programs including DOE algorithms can be applied to thermal-fluid problems. Local heat transfer coefficients have been determined by the variable groove diameters in streamwise direction and they can be easily examined with response surface method in ANSYS. We observe that the relation between local heat transfer coefficients and groove radii are nonlinear and designs can be optimized according to backward and forward effects to obtain the best local or total results. The circular grooves have not so much thermal effect at low Reynolds numbers $(<3000)$ since pseudo laminar behaviour of the flow. So, we obtained the maximum heat transfer coefficients in the case that the groove radii are minimum. However, when the Reynolds number increase thermal effects of grooves also increase thanks to turbulent flow and most efficient cases are obtained at higher groove radii. Since flow is turbulent, maximum heat transfer coefficients are obtained when the backward groove radii are maximum or forward groove radii are minimum. The hint is that we attained the local maximums when the own grooved section neither at minimum nor at maximum radius.

This study was supported by the Scientific Research Project Coordinatorship of Izmir Kâtip Çelebi University (2017ÖNAP-MÜMF-0005).

\section{References}

1. E.M. Sparrow, J.W. Comb, Int. Journal of Heat and Mass Transfer, 26, 7 (1983)

2. E.M. Sparrow, L.M. Hossfeld, Int. Journal of Heat and Mass Transfer, 27, 10 (1984)

3. P.G. Vicente, A. Garcia, A. Viedma, Int. Journal of Heat and Mass Transfer, 47, p. 671-681 (2004)

4. Y. Islamoğlu, C. Parmaksızoğlu, Applied Thermal Engineering, 23, p. 979-987 (2003)
5. Y. Islamoğlu, A. Kurt, Int. Journal of Heat and Mass Transfer, 47, p. 1361-1365 (2004)

6. Y. Islamoğlu, Int. Com. of Heat and Mass Transfer, 35, p. 643-647 (2008)

7. I. Taymaz, I. Koç, Y. Islamoğlu, Heat and Mass Transfer, 44, p. 1257-1262 (2008)

8. H. Pehlivan, Int. Journal of Heat and Mass Transfer, 66, p. 128-138 (2013)

9. K. Bilen, M. Çetin, H. Gul, T. Balta, Applied Thermal Engineering, 29, p. 753-761 (2009)

10. L. Wang, L. Deng, C. Ji, E. Liang, C. Wang, D. Che, Applied Energy, 174, p. 25-36 (2016)

11. J.A. Stasiek, Int. Journal of Heat and Mass Transfer, 41, p. 899-914 (1998)

12. L. Sun, C. Zhang, Int. Journal of Thermal Sciences, 75, p. 45-53 (2014)

13. H.Z. Han, B. Li, H. Wu, W. Shao, Int. Journal of Thermal Sciences, 90, p. 173-186 (2015)

14. W. Wang, Y. Zhang, Y. Li, H. Han, B. Li, Int. Journal of Heat and Mass Transfer, 120, p. 782-792 (2018)

15. S. Pethkool, S. Eimsa-ard, S. Kwankaomeng, P. Promvonge, Int. Com. in Heat and Mass Transfer, 38, p. 340-347 (2011)

16. S. Wang, G. Jian, J. Xiao, J. Wen, Z. Zhang, Applied Thermal Engineering, 119, p. 603-609 (2017)

17. A.F. Neto, A.F.B. Costa, M.F. Lima, Experimental Techniques, 42, 319-331 (2018)

18. B.C. Nookaraju, P.S.V. Kurmarao, S. Nagasarada, R. Karthikeyan, A. Vinay, Materials Today, 5, p. 52625271 (2018)

19. M. Cavazutti, Optimization Methods: From Theory to Design, Springer, (2013)

20. M. Savran, L. Aydin, Engineering Structures, Elsevier, 174, p. 675-687 (2018) 